\title{
Die gesellschaftlichen, infrastrukturellen und ökonomischen Folgen einer Pandemie
}

\author{
Wolf R. Dombrowsky
}

Über „Pandemie“ zu sprechen, sollte mehr einschließen als die Summe ihrer Teile. Von den Folgen her ist sie deutlich mehr und vor allem Anderes als mehrere nationale Epidemien zusammen. Je länger und „globalisierter“ die „Werkbänke“"1 sind, desto komplexer werden die dazu erforderlichen Austausch- und Steuerungsprozesse. In sie muss eine wirksame Pandemie-Bekämpfung eingreifen und genau daraus erwachsen Effekte, die über die unmittelbaren, nationalen Folgewirkungen des Epidemischen weit hinausgehen. Von daher greift jede Analyse zu kurz, die „Folgen“ nur im nationalen Kontext und anhand der Nahwirkungen des unmittelbar Epidemischen betrachtet. Weit folgenschwerer dürften die mittelbaren Folgewirkungen werden, die sich aus den „seuchenmedizinisch“ sinnvollen Interventionen als ökonomisch-technische „Side-Effects“ ergeben, so dass im Extrem dem Nutzen des medizinisch Sinnvollen weit größere, bislang noch nicht einbezogene „Kollateralschäden“ gegenüber stehen könnten. ${ }^{2}$

Entscheidend ist, was überhaupt als „Folgen“ gesehen und in abwägende Betrachtungen einbezogen wird. Bislang dominieren rein volks- und betriebswirtschaftliche Kalküle sowie „Zusammenbruchsadaptionen“ unterschiedlicher Herkünfte und Validität. Genannt werden Zusammenbrüche des Tourismus, des öffentlichen Verkehrs, der öffentlichen Versorgung, der Telekommunikation, der medizinischen und pflegerischen Versorgung, der Versorgung mit frischen Lebensmitteln und daraus folgend Zusammenbrüche von Ordnung und Disziplin samt „chaotischen Zuständen“. ${ }^{3}$ Unterschwellig bis offen werden die Szenarien der Versorgungs- und Funktionszusammenbrüche mit Zusammenbrüchen öffent-

1 Der Begriff entstammt der Produktionstheorie und meint die Auslagerung von Produktionsprozessen unter einem einheitlichen Management nach Maßgabe günstigster Faktorkosten (vgl. Zentes, J./Swoboda, B./Schramm-Klein, H.: Internationales Marketing, München 2006).

2 In der Medizin sind adverse Effekte seit langem bekannt. Ivan Illich kritisierte frühzeitig iatrogenetische Effekte der Medizin: Die Nemesis der Medizin. Die Kritik der Medikalisierung des Lebens. München: Beck 1995. Im Bereich der Industrieversicherung führten die Brände der Ersatzteil- und Logistikläger der Firmen Mitsubishi und Ford zu extremen Konflikten, weil die unmittelbaren Brandschäden nur einen Bruchteil der mittelbaren Ausfallkosten ausmachten, die zu monatelangen, globalen Lieferschwierigkeiten samt entsprechender Verluste führten. Dieser Zusammenhang ist haftungsrechtlich brisant und führt zu ökonomisch wie politisch delikaten Abwägungen.

3 So Lange, Werner: Pandemie-Szenarien, in: Bevölkerungsschutz 3/2007: 13-18 (16). 
licher Sicherheit und Ordnung und Übergängen zu Devianz und politischen Unruhen verquickt, ohne jedoch empirische Belege und deren Analysen beizubringen. Tatsächlich lässt sich für die gegenwärtigen Bedingungen über das erwartbare Verhalten von Individuen, Gruppen und Kollektiven nichts empirisch Fundiertes aussagen. Insbesondere die Worst-Case-Szenarien ermangeln übertragbarer Erfahrungen, doch sind generell alle szenarischen Folgenpfade von Randbedingungen umgeben, die empirisch nur sehr unvollständig untermauert und methodologisch nur sehr begrenzt modelliert werden können. Wer wird, bei einer Erkrankung von einem Drittel der Bevölkerung, ${ }^{4}$ seinen „Funktionen“ noch nachgehen können oder wollen, vor allem dann, wenn sich reale Betroffenheit im heimischen und das persönliche Risiko im sozialen Nahbereich häufen sollte? ${ }^{5}$

Vom Feldherrenhügel aus könnte sich eine umgekehrte Perspektive ergeben und daraus eine ebenso in die Irre leitende Fragestellung: Minderungen des BSP in Größenordnungen zwischen 1 und 8 Prozent zwingen keine Gesellschaft in die Knie; Mortalitätsraten bis 10 Prozent ebenso wenig. Selbst höhere Mortalitätsraten wie im Gefolge von Kriegen, ${ }^{6}$ wirken sich auf das Bevölkerungswachstum nur marginal ${ }^{7}$ und auf das Wirtschaftswachstum durchaus auch paradox aus. ${ }^{8}$ Letztlich geht es gar nicht um Bevölkerung als Abstraktum, sondern um eine Art „kritische Bestandsgröße“ in Bezug auf ihre grundsätzliche Überlebensfähigkeit.

Der Nationale Pandemieplan Deutschland (RKI) legt bei einer mittleren Erkrankungsrate von 30 Prozent etwa 103.000 Todesfälle zugrunde.

5 Der Reaktorunfall von TMI führte zur Diskussion des damals verstärkt auftretenden Phänomens von ,job-abandonment“, also dem absichtsvollen Fernbleiben von erwarteten und eingeplanten Funktionen. Die Angst, nach „Funktion“ in den Gefahrenbereich zu müssen, führte zu individuellen Eskapismusstrategien.

6 Schildt, Axel: Die Sozialgeschichte der Bundesrepublik Deutschland bis 1989/90. München: Oldenbourg Wissenschaftsverlag 2007:1-7 gibt einen Überblick über die Folgen des 2. WK für Deutschland. Im Dreißigjährigen Krieg kamen nach wiss. Schätzungen zwischen 10 und 25 Prozent der betroffenen Populationen um (vgl. Schmidt, Georg: Der Dreißigjährige Krieg. München: C. H. Beck 2003). Generell: Urlanis, Boris C.: Bilanz der Kriege. Die Menschenverluste Europas vom 17. Jahrhundert bis zur Gegenwart, Deutscher Verlag der Wissenschaften, Berlin 1965.

7 Dazu Eynern, Gert von: Zur Dynamik des Bevölkerungswachstums, Gewerkschaftliche Monatshefte 7/1956, H. 9, S. 552-561 (Auszug: http://library.fes.de/gmh/main/pdffiles/gmh/1956/1956-09-a-552.pdf) Aufschlussreich auch: Rainer, Bettina: Bevölkerungswachstum als Katastrophe. Apokalypse und Unsterblichkeit. Münster: Westfälisches Dampfboot 2004 (Diss. FU Berlin unter: http://www.diss.fu-berlin.de/diss/receive/ FUDISS_thesis_000000001024).

8 Krankheit und Tod sind wesentliche Wirtschaftszweige, Katastrophen wirken sich, neben allen Verlusten, durch Wiederaufbau und Ersatzinvestitionen, immer auch als Wachstumsimpuls aus. Rückversicherungen, Finanzprodukte wie Catbonds und Warenterminspekulationen auf Knappheiten zeigen zudem, dass Risiken und Risikoeintritte immer auch Anreize sind (vgl. Schiffmann, Olivia: Spekulieren auf die Katastrophe, http://emagazine.credit-suisse.com/app/article/index.cfm?fuseaction=OpenArticle\&aoid= 131287\&lang=DE). 
Auch dazu werden Untersuchungen angestellt, zumeist aber nicht veröffentlicht. Der produktive Apparat der Bundesrepublik Deutschland jedenfalls ließe sich im minimalen Extrem mit rund 17 Millionen Menschen aufrecht erhalten, ${ }^{9}$ was zu der vordergründig unethisch anmutenden Unterteilung nach „betriebswichtig“ und „,verzichtbar“ führt und der Frage, worum es beim „Seuchenschutz“ eigentlich geht? ${ }^{10}$

\section{Die gesellschaftlichen Folgen}

Von Island ließe sich lernen, worum es gehen könnte: 1783-85 brach die LakiSpalte aus, rund $20 \%$ der Bevölkerung fanden den Tod (vgl. Frömming 2006). Das damals zuständige dänische Ministerium erwog die vollständige Evakuierung und Aufgabe der Insel, weil das Königreich nicht bereit war, die Kosten eines Wiederaufbaus zu tragen. ${ }^{11}$ Die Isländer jedoch sprachen sich gegen eine Evakuierung aus ${ }^{12}$ und legten damit, ohne es damals absehen zu können, den Grundstein für ihre spätere unabhängige gesellschaftliche Entwicklung. ${ }^{13}$ Man entschied sich gegen die Aufgabe der Insel, aber mehr noch gegen ein Leben als „Einquartierte“ in einem fremden Land. ${ }^{14}$

92008 waren in Deutschland 40 Millionen erwerbstätig, 7,6 Millionen Menschen erhielten Leistungen der sozialen Mindestsicherung, davon 6,6 Millionen nach Hartz-IV, von denen wiederum $73 \%$ erwerbfähig waren und Arbeitslosengeld II bezogen.

10 Bei jeder gesellschaftlichen Notlage kehrt im Großen wieder, was der Katastrophenmedizin seit je originäre ethische Problemlage ist: Triage. Nach Maßgabe welcher Gründe werden knappe Rettungschancen wie verteilt? Hellsichtig dazu: Lübbe, Weyma (Hg.): Tödliche Entscheidung. Allokation von Leben und Tod in Zwangslagen. Paderborn: mentis Verlag 2004.

11 Während der Vulkantätigkeit vollzog sich in Dänemark ein krisenhafter Übergang. Die Regierungsgeschäfte übte anstelle des erkrankten Königs Christian VII. Staatsminister Ove Høegh-Guldberg aus, den jedoch Friedrich VI., Sohn Christians, dieser Aufgabe enthob.

12 Am 23. Januar 1973 brach auf Heimaey abermals ein neuer Vulkan aus, der „Feuerberg“ (Eldfell). Alle Insulaner wurden aufs eigene "Festland" (die Hauptinsel) evakuiert, niemand kam zu Schaden. Der Ausbruch dauerte fünf Monate, danach war die Insel um zwei Quadratkilometer größer. Rund 300 Häuser waren unter der Lava verschwunden, die Übrigen unter einer meterhohen Schicht aus Asche und Bimsstein. Die Regierung erklärte den nationalen Notstand, die Aufräumarbeiten dauerten fast zwei Jahre.

13 Island kam 1380 zusammen mit Norwegen unter dänische Herrschaft. Ureinwohner waren Kelten, Wikinger und Normannen (vgl. Thomasson, Richard F.: Iceland. The first new society. Minneapolis: University of Minnesota Press 1980). Klaus Schöter (Entstehung einer Gesellschaft. Fehde und Bündnis bei den Wikingern. Berlin: Reimer 1994) zeigt die Gründe, warum Island durchaus eine ganz eigene Gesellschaft darstellte.

14 Nach dem 2. Weltkrieg erlebten auch Millionen Deutsche dieses Gefühl, teilweise über mehrere Generation hinweg (vgl. Kossert, Andreas: Kalte Heimat. Die Geschichte der deutschen Vertriebenen nach 1945. München: Siedler 2008; Radebold, Hartmut/ 
Auch ganz Anderes wäre denkbar, sozusagen ein generalisiertes Radevormwald: Am 27. Mai 1971 fuhren ein Güterzug und ein Schienenbus auf eingleisiger Strecke bei Dahlerau frontal ineinander. 61 Schüler der Abschlussklasse der Geschwister-Scholl-Schule, 5 Lehrer und eine begleitende Mutter kamen dabei ums Leben. Letztlich war jede Familie in Radevormwald vom unzeitigen Tod betroffen und über Jahrzehnte hin traumatisiert. Radevormwald erschien lange Jahre wie gelähmt und in Trauer verharrt. Nichts wünschten sich die Betroffenen sehnlicher, als das Geschehene ungeschehen machen zu können.

Was also unter welchen Bedingungen „lebens-“ oder „erhaltenswert“ erscheint, ergibt sich aus den situativen Konstellationen und dem Abgleich mit anderen Werten, ${ }^{15}$ am wenigsten aber aus der Extrapolation rein ökonomischer Indikatoren. Gerade derer aber bedienen wir uns in erster Linie, um die Folgen einer Pandemie abschätzen zu wollen. Dabei geht es nicht zuvörderst um Probleme der Bewertbarkeit, also der Frage wie menschliche Erlebensqualitäten und handlungsleitende Einstellungen quantifiziert werden können, sondern um die Erfassung und Abschätzung dieser Qualitäten selbst. Tatsächlich besteht hier das größte Desiderat: Was sind überhaupt „gesellschaftliche“ Folgen und wie erheben wir sie empirisch? Die Übertragung eines generalisierten Radevormwalds auf eine von einer Pandemie geschlagene Bundesrepublik führt zu einem eigentümlichen Szenarium, sozusagen zu einer traumatisierten, trauernden wie traurigen, vielleicht gar depressiven Republik, die aber wie Radevormwald nach dem Unglück, dennoch dem Alltäglichen nachgeht. Die Menschen in all ihrem Unglück stellen nicht das Leben ein, auch wenn sie es unglücklich führen. Insofern „funktionierte“ die Bundesrepublik als Gesellschaft unbeschadet weiter und sie könnte dies durchaus auch mit zwei Drittel ihrer gegenwärtigen Einwohner oder sogar mit der szenarisch-gespenstischen Minimalbesatzung von 17 Millionen Arbeitenden.

Im Falle der Evakuierung der Isländer nach Dänemark wäre dies nicht der Fall gewesen; sie hätte das Ende der isländischen Gesellschaft und damit vermutlich

Bohleber, Werner/Zinnecker, Jürgen: Transgenerationale Weitergabe kriegsbelasteter Kindheiten: Interdisziplinäre Studien zur Nachhaltigkeit historischer Erfahrungen über vier Generationen. München: Juventa 2007; Bode, Sabine: Die Kriegsenkel: Die Erben der vergessenen Generation. Stuttgart: Klett Cotta 2010). Eine generelle Analyse legt Hartmut Böhme vor: Gewalt im 20. Jahrhundert. Demozide in der Sicht von Erinnerungsliteratur, Statistik und qualitativer Sozialanalyse, figurationen 0 (1999), S. 139-157.

Die Dürftigkeit dieser Formulierung im Angesicht von Schmerz und Leid ist offensichtlich, gleichwohl generell nur schwer zu erhellen. Persönlich hänge ich der Feldtheorie von Kurt Lewin an: Feldtheorie in den Sozialwissenschaften. Bern: Huber 1963, die Erhellendes beiträgt, wenn es um das Zustandekommen von persönlichen, psychischen „Haltungen“ geht, auf deren Basis mit Bedrückendem umgegangen werden kann. Ganz wesentliche Aufschlüsse geben Kloepfer, Michael/Deye, Sandra: Pandemien als Herausforderung für die Rechtsordnung, DVB1. 2009 (Okt.): 1208-1221. 
auch das Ende der Isländer bedeutet, die nicht als „Isländer“, sondern als Untertanen des dänischen Königs behandelt worden wären. Wie die rund 55.000 Isländer aber tatsächlich auf die rund 840.000 dänischen Einwohner ${ }^{16}$ verteilt worden wären und wie sie sich vertragen hätten, vermag niemand zu sagen, wäre aber das eigentlich Bedeutsame in Bezug auf gesellschaftliche Folgen.

So besehen wird zumindest deutlich, dass ein Zusammenbruch von Gesellschaft nicht durch eine Epidemie/Pandemie herbeigeführt werden kann, sondern nur durch die Aufgabe oder Auflösung von „Staat“ und „Staatsvolk“. Bleibt das Territorium erhalten, hat auch Gesellschaft Bestand, unbeschadet des nachfolgenden „Überlebensgefühls“ und der Reorganisation des Gesellschaftlichen nach Maßgabe seiner realen zahlenmäßigen Zusammenhänge. Entscheidend bleibt die Demographie, weswegen es bedeutsam ist, welche Altersgruppe mehr und welche weniger gefährdet erscheint und welche Strategie zur Minderung dieser Gefährdung anempfohlen wird. ${ }^{17}$

Entlang dieser Demographie verteilen sich auch Besorgnis und Sorgebedarf. Sind eher Kinder und Jugendliche betroffen, wird dies die elterliche Sorge beanspruchen und isolierende Strategien in Richtung Familie hervorrufen, sind eher Ältere betroffen, wird dies den Pflege- und Versorgungsbedarf erhöhen und eher aufsuchende Dienstleistungen beanspruchen. In beiden Fällen ist mit zusätzlichen Belastungen des Gesundheitssystems und der Versorgungsdienste zu rechnen, vor allem dann, wenn die Pandemie/Epidemie in mehreren Wellen verlaufen sollte. Von daher wird es erforderlich, Ressourcen verfügbar zu machen, um Ausfälle kompensieren zu können. Dies dürfte all jenen eher gelingen, die sich aus Vorsicht zwar selbst isolieren, aber dennoch eine geeignete Allokation externer Ressourcen zu organisieren vermögen. Personen, die dagegen ohnehin isoliert leben, sind strukturell im Nachteil. Sie verfügen weder über eigene Ressourcen noch über kompensierende Netzwerke, die sie mobilisieren, sobald die Netze aufsuchender Dienste ausfallen.

16 Die erste dänische Volkszählung fand 1769 statt, allerdings gingen die meisten Daten verloren, weswegen die Erhebung von 1787 als die „eigentliche“ gilt. Stand damals: 841.806 Einwohner. Die erste isländische Volkszählung (Manntalið prjú hundruð ara) fand bereits 1703 statt.

17 In jedem Pandemieplan finden sich Angaben zu den gefährdeten Alterskohorten und zu einer darauf bezogenen Impfstrategie. Letztlich re-biologisieren hier alle soziokulturellen Erwägungen, weil es keinen Sinn macht, bei höchst wahrscheinlicher Gefährdung der Jungen, auch die Alten zu impfen und umgekehrt. Viel bedenklicher sind dagegen Gleichverteilungserwartungen: In welcher Reihenfolge impft man bei Knappheit an Impfstoff oder Personal bei altersunabhängiger Mortalitätserwartung? 
Schon ein Vergleich der szenarischen Erkrankungsraten zeigt, ${ }^{18}$ dass es unbedingt kompensierender Ressourcen bedarf, weil bereits das System der gesundheitlichen Allgemeinversorgung nicht mehr funktionieren kann:

\begin{tabular}{|c|c|c|c|}
\hline Erkrankungsrate & $\begin{array}{l}\text { Zusätzliche } \\
\text { Arztbesuche }\end{array}$ & Krankenhauseinweisungen & Todesfälle \\
\hline 15 Prozent & $6,5 \mathrm{Mio}$ & 187.000 & 51.460 \\
\hline 30 Prozent & $13,1 \mathrm{Mio}$ & 374.116 & 102.920 \\
\hline 50 Prozent & $21,8 \mathrm{Mio}$ & 623.527 & 171.533 \\
\hline
\end{tabular}

$\mathrm{Zu}$ den anderen Versorgungssystemen existieren keine entsprechenden Abschätzungen. Nicht nur die niedergelassenen Ärzte und Krankenhäuser wären überfordert, sondern auch Apotheken und Pflegedienste, ${ }^{19}$ Bestattungsinstitute und Friedhofsverwaltungen, Krankentransport- und Taxiunternehmen, Bringdienste („Essen auf Rädern“) und andere ,aufsuchende Hilfen“. Die Frage wäre, ob und wie sich eine Gesellschaft, der die laienmedizinische Selbstversorgungsfähigkeit abhanden gekommen ist, medizinisch helfen könnte, sobald deren professionalisierte Medizin ihre Hilfsversprechen nicht mehr einzulösen vermag. Nach Planungslage sind die Dienstpersonale der Gesundheitsversorgung ,systemrelevant“ und erhalten bevorrechtigt Leistungen zur Aufrechterhaltung ihrer Funktionsfähigkeit. Allerdings zeigte sich im Zuge von Pocken- und Schweinegrippenimpfungen, dass vor allem die medizinnahen Personale größte Reserven an den Tag legten. Eine auf Aufrechterhaltung von Systemfunktion abzielende Planung müsste deshalb vor allem Maßnahmen entwickeln, die „Entsatz“ oder BypassKapazität eröffnen, um der strukturell doppelten Abhängigkeit von Mangelkapazität durch epidemische Ausdünnung und ,functional abandonment“ innerhalb der funktionalen Rest-Kapazität zu entgehen. Bislang jedoch werden Formen einer „epidemischen Barfuss-Medizin“ oder einer häuslichen und nachbarschaftlichen Laien-Medizin nicht einmal im Ansatz gedacht. Noch ferner scheinen Konzepte zu liegen, die den Gedanken der Kompensation zum Ausgang nehmen und Verfahren ersinnen, wie ein Depot- und Konsultationssystem auszusehen hätte, durch das sich die (selbst-) quarantänisierte Bevölkerung Rat und materiale Hilfe holen könnte.

18 Es liegen die Zahlen zugrunde, wie sie Martin I. Meltzer, Nancy J. Fox und Keiji Fukuda: Economic Impact of Pandemic Influenza in the United States: Priorities for Intervention, Emerging Infectious Diseases 5, 1999,5: 659-671 und der Nationale Pandemieplan des RKI verwendeten.

19 Von den hospitalisierten Erkrankten werden wahrscheinlich 15-20 Prozent intensiv gepflegt und etwa die Hälfte beatmet werden müssen. 
Die in zwei Wellen verlaufene „Honkong-Grippe“ von 1968/69 und 1969/70 zeigte jedenfalls, zu welchen Engpässen und Zusammenbrüchen es bei einer größeren Pandemie kommen kann: So stand für die 2. Welle nicht genug Impfstoff zur Verfügung, erließen die Kliniken Aufnahmestopps, standen nicht mehr genug niedergelassene Arzte für eine flächendeckende Versorgung zur Verfügung, fehlten die Kapazitäten zur Beerdigung und vielfach die Personale für Notfälle (Feuerwehr, Polizei, Rettungsdienst). Erste Engpässe in der Produktion und Distribution traten auf und damit Versorgungsschwierigkeiten bei Lebensmitteln.

Schon aus vorbeugender Sicht wird es nötig sein, möglichst alle Gelegenheiten zu erhöhter sozialer Dichte zu unterbinden, wodurch nicht nur der gesamte Sport-, Freizeit-, Kultur- und Bildungsbereich zum erliegen käme, sondern auch Märkte und Einkaufszentren, Bahnhöfe und Postämter, Banken und Behörden. Um dennoch grundlegend erforderliche Angebote aufrecht zu erhalten, wäre ein Balancement aus Bedarf und Infektionskontrolle erforderlich, für das es bislang kein Antezedenz gibt. Wie verteilt eine Gesellschaft Waren und Dienste, wenn die üblichen Zentren der Verteilung notwendig Horte der Ansteckungsgefahr sind? Und wie organisiert eine Gesellschaft ihre Verteilung um, wenn diese einerseits auf Just-In-Time-Strukturen und andererseits auf individueller Mobilität beruht? ${ }^{20}$ Auf dieser Organisationsstufe reichen die Strategien der Kompensation nicht mehr hin, zumal sie ohnehin nur für mikrosoziale Aggregate (Individuen, Gruppen, Familien, Nachbarschaft) taugen. Auf meso- und makrosozialer Ebene bedarf es geplanter und organisierter Entsatzstrategien und damit der Entsatzinfrastrukturen.

\section{Die infrastrukturellen Folgen}

Bis zu einem gewissen Grade ergibt sich gesellschaftliche Verletzlichkeit als nicht intendierte, aber gleichwohl unvermeidbare Nebenfolge des primär Gewollten: Wenn aus Gründen betriebswirtschaftlicher Rationalität auch in dem Sinne ,verlängerte Werkbänke“ entstehen, dass man die Lagerhaltung ,mobilisiert“", ${ }^{21}$ wird ganz buchstäblich die gesellschaftliche Verkehrsinfrastruktur zum volkswirtschaftlichen Gesamtlager, das seine Verfügbarkeit verliert, sobald der Verkehr oder die zur Lagerdisposition nötige Steuerung ins Stocken geraten. Die dann irgendwo liegen gebliebenen Lager bekommen „Strandgut"“-Charakter (und

20 Die Tengelmann-Gruppe legt ihren Szenarien eine Versorgungsreserve von 2,6 - 1,3 Tage zugrunde, danach sind die Läden leer.

21 Probleme, Vor- und Nachteile beschreiben Seuring, Stefan/Müller, Martin/Goldbach, Maria/Schneidewind, Uwe (eds.): Strategy and Organization in Supply Chains. Heidelberg, New York: Physica 2003. 
werden gelegentlich so behandelt) oder sie werden selbst zur Gefährdung, die spezifischer Sicherung, Bergung und Entsorgung bedarf. ${ }^{22}$

Bereits das simple Beispiel zeigt die Brisanz von Interdependenzen, die während ihres reibungslosen Funktionierens als Produktivitätsfortschritt gefeiert werden, aber ihre Janusköpfigkeit zeigen, sobald das filigrane Räderwerk zu stocken beginnt. Weit stärker noch als an der Verkehrsinfrastruktur zeigte sich diese Janusköpfigkeit bei Zusammenhängen, die verschiedene Infrastrukturen zu komplexeren „Megastrukturen“ kombinierten. Als im Zuge so genannter Marktliberalisierung Stromerzeugung und -verteilung entkoppelt und die dann selbständigen Systeme betriebswirtschaftlich optimiert wurden, ergaben sich ganz zwangsläufig nicht intendierte gesellschaftliche Schwächungseffekte. ${ }^{23}$ So lassen sich die Blackouts in den USA und in Europa auch darauf zurückführen, dass die betriebswirtschaftlich erstrebenswerte Auslastung von Leitungskapazitäten umgekehrt auch dazu führt, dass im Notfall keinerlei Reserven verfügbar sind, mittels derer Überkapazitäten abgeführt oder Unterkapazitäten überbrückt werden können. Völlig analoge Effekte ergeben sich aus der betriebswirtschaftlichen Optimierung der Krankenhausversorgung. Auch sie führt zu einer ständigen Auslastung der Bettenkapazität und damit, nicht intendiert aber folgerichtig, zur völligen Unterversorgung bei jedem Massenanfall von Verletzten.

Insbesondere die Ökonomie des Gesundheitswesens wird sich im Falle einer Pandemie dramatisch auswirken, auch weil sich die durchschnittliche Verweildauer der Patienten in den letzten zehn Jahren von 13 auf 8 Tage verringert hat, so dass eine Kapazitätserhöhung durch vorzeitige Entlassungen medizinisch kaum mehr vertretbar und zudem epidemiologisch riskant wäre, weil eine Entlassung Rekonvaleszenter in die häusliche Pflege nicht nur deren Infektionsrisiko erhöhte, sondern auch die Kompensationsfähigkeit der aufnehmenden Verbände minderte.

Das Beispiel verweist zugleich auf das Grundsätzliche. Ausfälle von komplexen Infrastrukturleistungen bedürften hoher Absicherungsleistungen für Überbrückung, Kompensation und Entsatz. ${ }^{24}$ Am häufigsten werden sie durch erhöhten

22 Insbesondere beim Ausfall von Kühlketten (Lebens- und Arzneimittel, Blutkonserven) oder beim Transport lebender Tiere zeigen sich massive Probleme.

23 Vgl. Joskow, Paul L.: California's Electricity Crisis, Oxford Review of Economic Policy 17, 2001, 3: 365-388.

24 Von Sicherstellungsmaßnahmen bis zu redundanten Leistungsvorhaltungen steht jeder Gesellschaft ein Spektrum an Möglichkeiten zur Verfügung. Einzigartig dürften allerdings die Notfallversorgungsregelungen von Singapur sein. Der Stadtstaat verpflichtet sämtliche Lieferanten versorgungsrelevanter Leistungen zu einer Notversorgungsgarantie selbst für Kriegsfälle von mindestens 6 Monaten. Für Güter wie Ö1, Gas und Wasser gelten spezifische Regelungen. Beispielhaft: World Bank AAA Program: Dealing with Water Scarcity in Singapore: Institutions, Strategies, and Enforcement. Washington D.C. 2006 . 
Einsatz menschlicher Leistungen kompensiert. So wurde während der Rheinverschmutzung durch den Brand der Firma Sandoz in Schweitzerhalle in einigen Kommunen die Trinkwasserversorgung durch Tankwagen sichergestellt, von denen sich die Einwohner ihren Tagesbedarf mit Kanistern selbst abholten. Fraglich ist jedoch, ob unter den Ausfallbedingungen einer Pandemie solche Kompensationsleistungen überhaupt leistbar wären und wie man sie zudem zeitlich und räumlich staffeln müsste, damit keine infektionsfähigen Dichten entstehen können.

Die zentrale Herausforderung einer Pandemie besteht somit darin, dass die ansonsten bei Notfällen und Katastrophen wirksame Entsatzstrategie „Soziales statt Technik“ entweder durch krankheitsbedingte Ausfälle gar nicht in Anschlag gebracht werden kann oder so modifiziert werden muss, dass sie in erster Linie den Bedingungen der Infektionsunterbrechung zu gehorchen hat. Eine solche Sichtung und Bewertung von Infrastrukturleistungen nach Kompensationsvermögen steht jedoch noch aus. Sie aber wäre die Grundlage für die Planung resilienter Entsatzinfrastrukturen.

Grundsätzlich belässt eine Pandemie die grundlegenden Infrastrukturen im technischen Sinne intakt. Infrastrukturausfälle ergeben sich ausschließlich aus den Ausfällen personaler Ressourcen. So gesehen ist das Attribut „resilient“ für Infrastrukturen irreführend, weil nicht die Infrastrukturen zu härten wären, sondern die sie bedienenden Personale. Von daher ist die bevorzugte Impfung betriebs- und versorgungswichtiger Personale unverrückbare Grundbedingung. Sie bedarf aber einer Weiterentwicklung in Richtung struktureller Quarantänisierung und quarantänisierter Mobilität, um zum einen das Funktionspersonal unverzichtbarer Infrastrukturen über längere Zeiträume autark handlungsfähig zu machen und um zum anderen Dienste und Leistungen ohne Infektionsrisiko zu den Isolationsinseln der Bevölkerung entsenden oder „Besorger“ aus der Bevölkerung so schützen zu können, dass diese als Transportkapazität fungieren könnten. Aus allem ergäben sich Strategien des infrastrukturellen Entsatzes.

\section{Die ökonomischen Folgen}

Ein Report der Allianz ${ }^{25}$ fasst die Problematik ökonomischer Folgen in drei Kapiteln so zusammen: „Infiziert das Virus auch das Bruttoinlandsprodukt?“ (3846), „Wie preist der Kapitalmarkt den Erreger ein?“ (47-51) und „Wen macht das Virus zu Verlieren, wen zu Gewinnern?““(52-61). Die Antworten beruhen 
allesamt auf Zusammenstellungen von Abschätzungen, die Analysten von Banken, Versicherungen, Unternehmen und Behörden vorgenommen haben.

Das daraus entstehende Bild ist relativ monochrom, sofern man die Unterschiede der nationalen Gesundheits- und Lebenshaltungskosten berücksichtigte: Die australische Regierung legt allein ein gravierendes Szenario mit einem BSPRückgang von 5\% zugrunde. ${ }^{26}$ Die Studie des „Congressional Budget Office“ der US-Regierung beschreibt für die USA eine milde Variante mit einem Rückgang des BSP um 1,5\% und eine gravierendere Variante mit einem Rückgang um 5\% im Jahr der Influenza-Pandemie. Für Europa geht die Studie des Lowy Institute von einem Rückgang des BSP zwischen $0,7 \%$ und $8 \%$ aus, während die Schweizer Studie ${ }^{27}$ rund 400 Mio. CHF $(0,13 \%)$ an direkten Gesundheitskosten und rund 1.9 Mrd. (0,6\%) an indirekten Folgekosten veranschlagt, bezogen auf das BSP von 2008 (317 Mrd. CHF). Was sagen nun diese Rückgänge und womit ließen sie sich vergleichen? Der Weltbankökonom Milan Brahmbhatt schätzt den globalen Gesamtverlust durch eine Pandemie auf 683 Mrd. Euro, der Internationale Währungsfonds beziffert die Verluste durch die Finanzkrise auf rund 603 Mrd. Euro, ${ }^{28}$ worauf „die Märkte“, so N24, „gelassen reagierten“. Warum also sollten die Märkte auf die Folgen einer Pandemie weniger gelassen reagieren, zumal es sich ja weitgehend um suspendierende Effekte handeln dürfte, also um saisonale Ausfälle von Handlungen (z. B. Reisen oder privaten Konsum), die, nach dem Abklingen der Pandemie, mit großer Wahrscheinlichkeit nachgeholt werden. Anders als bei der Finanzkrise handelt es sich in den meisten Fällen um Effekte innerhalb der Realwirtschaft und um Ausgabenausfälle, ohne Verlust der Ausgabenfähigkeit.

Zudem lässt sich vermuten, dass aufgrund einer Pandemie enorme Wachstumsimpulse eintreten, sobald die Pandemie vorüber ist. Dies gilt nicht nur für Konsumeffekte im Erlebnisbereich, sondern auch überall dort, wo Versicherungsleistungen fällig werden und dadurch erweiterte Kaufkraft und Investitionsfähigkeit verfügbar werden. Dies wiederum führt zu erhöhtem Steueraufkommen und damit zu einer Erweiterung des staatlichen Entscheidungsspielraums, auch hin zu fördernder Staatstätigkeit. Die hohe Sterblichkeitsrate der Pandemie führt, je nach Altersverteilung, zu Verknappungseffekten, unter Umständen an Arbeitskräften, so dass dies ein steigendes Lohnniveau bedingte, wodurch wiede-

26 Tatsächlich liegen Bankdaten zugrunde: HSBC: Assessing the economic impact of a flu pandemic, Australia and New Zealand Weekly, Jan. 9, 2006: 1-4.

27 Im Auftrag des Bundesamtes für Gesundheit (BAG) mit Unterstützung des Bundesamtes für Bevölkerungsschutz (BABS): http://www.bag.admin.ch/themen/medizin/00682/00686/ 02314/index.html?lang=de.

28 Zit. nach N24 online (http://www.n24.de/news/newsitem_650242.html). 
rum die Kaufkraft und der Konsum wüchsen, so dass insgesamt eine Pandemie zu massivem Wirtschaftswachstum führen könnte.

Selbstverständlich ergäben sich global wie sektoral höchst unterschiedliche Folgewirkungen. Schon heute ,preisen“ vor allem Versicherungen die zu erwartenden Leistungspflichten ein und erhöhen die Prämien oder entwickeln neue Versicherungsprodukte, um eine bessere Risikostreuung zu erreichen. Auch der Pharma-, Gesundheits-, Diagnose- und Schutzbereich profitierte von Pandemien wie auch der Pandemieprävention. Ebenfalls rechnen alle Experten mit deutlich unterschiedlichen Auswirkungen auf Industrienationen, Schwellenländer und weniger entwickelte Ökonomien. Wie auch bei anderen Katastrophen führen auch Epidemien/Pandemien dazu, dass die wohlhabenden Volkswirtschaften die Schäden leichter verkraften können als die armen, vor allem auch deshalb, weil dort Gesundheitskosten besonders zu Buche schlagen. Sofern ohnehin die Mittel für Gesundheitsausgaben fehlen, könnte sich dies im Pandemiefall auch dahingehend auswirken, dass sich, umgekehrt proportional zum Prokopfeinkommen die Zahl der Todesfälle erhöht. Dies wiederum hätte Auswirkungen auf die Nachfrage nach Lebensmitteln, Düngemitteln und Treibstoffen und führte zu deren Verbilligung.

$\mathrm{Zu}$ beachten ist natürlich, dass alle Abschätzungen auf Modellen beruhen. ${ }^{29}$ Letztlich handelt es sich immer um Aggregierungen von Einzelschäden. Häufigkeit wie Verteilung dieser Schäden variieren jedoch stark, oftmals fehlt es an verlässlichen oder übertragbaren Daten sowie an brauchbaren Abschätzungen der Eintrittswahrscheinlichkeiten. Deshalb werden zumeist stochastische Modellierungen mehrdimensionaler Daten verwendet ${ }^{30}$ oder einfach historische Beispiele hochgerechnet. Letzteres gilt vor allem für die Abschätzung der Mortalität, wobei die Problematik der Berechnung und Berechenbarkeit seltener Ereignisse noch immer ungelöst ist. ${ }^{31}$ Ebenfalls problematisch bleibt die Transformation nicht markterfasster Güter und Dienstleistungen in monetär bewertete Einheiten sowie die Prognose von substituierenden Handlungen im Gefolge einer Pandemie. Dies gilt vor allem für Effekte von Tauschbeziehungen über graue oder schwarze Märkte sowie die dafür erforderliche Erstellung von Gütern und Leistungen. „Hamsterfahrten“ wie nach Kriegen oder „Plünderungszüge“ ganzer

29 Die beste Zusammenstellung relevanter und kommentierter Literatur zur Modellentwicklung gibt Müller, Roland: Modellgeschichte ist Kulturgeschichte. Eine Chronik von Modellgebrauch und Modellbegriff. Ein Literaturbericht.

30 Dazu Ender, P.: Stochastische Modellierung von Katastrophen-Schäden.

31 Generell gibt es kein systematisches Verfahren, „mit dem man testen kann, ob ein Modell alle Systemzustände erfasst, die für das erwünschte Ereignis relevant sind“, so Tittes, Eberhard: Zur Problematik der Wahrscheinlichkeitsberechnung bei seltenen Ereignissen, in: Compes, Peter (Hrsg.): Technische Risiken in der Industriegesellschaft. Erfassung Bewertung - Kontrolle. Wuppertal 1986: 345-372 (350). 
Kollektive oder Stadtteile wie im Gefolge spezifischer Katastrophen kollidieren mit berechtigten Infektionsängsten, so dass hier keine Analogieschlüsse möglich sind. $^{32}$ Von daher könnten Pandemien reale „Fallen-Ereignisse“ sein: Die Betroffenen sitzen, aufgrund ihrer eigenen Ansteckungsängste, aber mehr noch aufgrund der seuchenhygienischen Unterbrechungsmaßnahmen buchstäblich in der Falle totaler Isolation. Während alle anderen katastrophalen Zusammenbrüche durch ad-hoc-Kooperation sektoral kompensiert werden können, stehen diese „emerging ressources“ im Pandemiefall nicht zur Verfügung. Dies wird die eigentliche Katastrophe werden. Sie ließe sich nur vermeiden wenn es gelänge, die emerging ressources gegenüber dem Ansteckungsrisiko zu immunisieren, zumindest zu schützen, damit sich die Ausfälle sozial kompensieren und überbrücken lassen. nungspolitische Chiffre. Notfallvorsorge 3/2005: 8-11. 